\title{
Platelet poor plasma gel combined with amnion improves the therapeutic effects of human umbilical cord-derived mesenchymal stem cells on wound healing in rats
}

\author{
LUJUN YANG ${ }^{1,2^{*}}$, JIE MA $^{1,2^{*}}$, SAIYANG GAN ${ }^{1}$, SHULI CHU ${ }^{1}$, \\ MARTIN MALDONADO ${ }^{2}$, JIANDA ZHOU ${ }^{3}$, LIAN MA ${ }^{2}$ and SHIJIE TANG ${ }^{1}$ \\ ${ }^{1}$ Department of Burns and Plastic Surgery, The Second Affiliated Hospital, Shantou University Medical College; ${ }^{2}$ Research \\ Center for Translational Medicine, Shantou University Medical College, Shantou, Guangdong 515041; ${ }^{3}$ Department \\ of Plastic Surgery, The Third Xiangya Hospital, Central South University, Changsha, Hunan 410013, P.R. China
}

Received August 8, 2016; Accepted May 8, 2017

DOI: $10.3892 / \mathrm{mmr} .2017 .6961$

\begin{abstract}
The aim of the present study was to investigate the efficacy of human umbilical cord-derived mesenchymal stem cell (HUMSCs) embedded in platelet poor plasma (PPP) gel combined with amnion (PPPA) in improving wound healing on Sprague-Dawley (SD) rats. HUMSCs were cultured and labeled with chloromethylbenzamido-1,1'-dioctadecyl-3,3,3'3'-tetramethylindocarbocyanine perchlorate (CM-DiI) on their third passage. The expression levels of growth factors of HUMSCs in PPPA were assessed by ELISA. Full-thickness excisional skin wounds were induced in 36 male SD rats, which were treated with PPPA grafted with HUMSCs (PPPAC), PPPA, or HUMSC or PBS injection. The degree of healing and the distribution of labeled HUMSCs in the wound were evaluated by hematoxylin and eosin (H\&E) staining and immunofluorescence. On day 14 post-surgery, wound healing in PPPAC-treated rats was significantly higher than the PPPA group, compared with rats treated with HUMSCs alone and control rats $(\mathrm{P}<0.05$ and $\mathrm{P}<0.01$, respectively). $\mathrm{H} \& \mathrm{E}$ staining showed that morphology and thickness of the epidermis in the PPPAC group was similar to that of healthy skin. ELISA revealed that levels of growth factors of HUMSCs in PPPAC were higher than in monolayer cells. In conclusion, PPPA can modify growth factor expression levels of HUMSCs and improve the efficiency of HUMSCs in the healing of full thickness wounds in rats.
\end{abstract}

Correspondence to: Dr Lujun Yang, Department of Burns and Plastic Surgery, The Second Affiliated Hospital, Shantou University Medical College, 69 Dongxia Road, Shantou, Guangdong 515041, P.R. China

E-mail: yanglujun726@hotmail.com

*Contributed equally

Key words: human umbilical cord-derived mesenchymal stem cell, platelet poor plasma gel, cell growth factors, full-thickness excisional skin wounds, wound healing

\section{Introduction}

Chronic and non-healing wounds give rise to significant morbidity and mortality, as well as considerable health care expenditure. Devising a therapeutic approach in order to treat extensive deep wounds remains a major clinical challenge (1). Due to the lack of effective treatments, a combination of conventional managements such as surgical debridement and skin grafting with skin substitutes in combination with growth factors have been used to treat wounds. The potential application of mesenchymal stem cells (MSCs) in accelerating wound healing has been an active area of investigation (2).

MSCs were first identified in bone marrow (3), but it has since been subsequently demonstrated that MSCs are present in nearly all tissues (4). However, MSCs isolated from bone marrow, adult organs and fetuses exhibit several disadvantages, including invasive methods for isolation, low yield and a variety of ethical issues. Stem cells from other sources such as embryonic stem cells (ESCs) and induced pluripotent stem cells (iPSCs) also have clinical concerns, including potential immunorejection and/or tumorigenesis (5). Therefore, there is the need to identify additional sources for the collection of MSCs that do not have the above mentioned technical, ethical and clinical concerns. The umbilical cord is regarded as a promising source of MSCs. As perinatal stem cells, human umbilical cord-derived mesenchymal stem cells (HUMSCs) have the potential for self-renewal, extended proliferation and immunomodulatory properties, making them excellent candidates for stem cell-based therapy (6). HUMSCs also possess characteristics of stem cells, such as multipotency and the ability to differentiate into cells of multiple lineages, such as bone, osteoblasts, chondroblast and adipocytes, under appropriate differentiation conditions $(7,8)$. As HUMSCs are obtained from discarded umbilical cord tissue during caesarean sections, there are fewer ethical issues involved compared with ESCs. Compared with MSCs derived from adipose and bone marrow, HUMSCs represent a source of more primitive stromal cells that do not undergo spontaneous malignant transformation (9). Together with their relative ease of accessibility, cost-effectiveness, and efficacy, it is predicted 
that HUMSCs will have increasing application in tissue engineering and cellular-based therapies (10).

MSCs contribute to wound repair through several mechanisms including: i) structural repair of wounds via differentiation; ii) immunomodulation; iii) paracrine release of growth factors that promote neovascularization and re-epithelialization; and iv) mobilization of the resident stem cell niche (11). Among these mechanisms, it is widely believed that MSC paracrine signaling is the predominant mechanism accounting for enhanced wound repair and reduced scar formation (12-14). However, paracrine activities are influenced by many factors, including the local microenvironment and cell culture conditions. Traditionally, HUMSCs are commonly cultured as 2-dimensional monolayers on tissue culture plastic. This method is widely regarded as poorly efficient, as evidenced by the poor survival of HUMSCs engrafted at the site of injury. In addition, in vivo, HUMSCs support the extracellular matrix. Therefore, HUMSCs are integrated in a 3-dimensional manner in vivo once grafted. Optimization of culture conditions for HUMSCs to achieve successful engraftment remains an area of active research.

The present study mimicked the 3-dimensional in vivo environment by culturing HUMSCs with platelet poor plasma (PPP) gel combined with amnion (PPPA) to evaluate the influence of a new carrier, PPPA, on the healing efficacy in treatment of full-thickness excisional skin wounds in Sprague-Dawley rats. ELISA was also performed to assess the expression of HUMSC growth factors in a variety of experimental conditions and timings in vitro. The aim of the present study was to evaluate the potential of a novel carrier for HUMSCs in wound healing in vivo.

\section{Materials and methods}

Umbilical cords and amnion collection. Umbilical cords and amnion from four full-term (38-40 weeks) healthy pregnancies (excluding HIV, hepatitis and syphilis) through cesarean section were obtained from the Department of Obstetrics and Gynecology, the Second Affiliated Hospital of Shantou University Medical College (Shantou, China). Informed consent and ethical permission were obtained before parturition in accordance with the Ethics Committee of Shantou University Medical College.

Isolation, culture and identification of HUMSCs from human umbilical cords. Isolation, culture and identification of HUMSCs was performed in the translational medicine center, as previously described (15). Briefly, within $24 \mathrm{~h}$ after collection of umbilical cords from the operating room, umbilical cords were flushed with phosphate buffered solution (PBS) to remove blood cells. Umbilical cords containing two arteries and a vein buried within the mucous connective tissue, known as the Wharton's jelly, were sectioned into pieces $(3-4 \mathrm{~cm}$ long) with a sterile scalpel. After removing intact vessels, the remaining Wharton's jelly was transferred to a sterile container containing high glucose Dulbecco's modified Eagle's medium (H-DMEM; Gibco; Thermo Fisher Scientific, Inc., Waltham, MA, USA) and then dissected into smaller pieces. Thereafter, explants were plated on $100 \mathrm{~mm}$ cell culture dishes (JET BIOFIL) which were inverted in a humidified atmosphere with
$5 \% \mathrm{CO}_{2}$ at $37^{\circ} \mathrm{C}$ for $15-30$ min until the explants adhered to the bottom of the dish. Fresh growth medium $55 \mathrm{ml}$; H-DMEM supplemented with $10 \%$ fetal bovine serum, $100 \mathrm{mg} / \mathrm{ml}$ penicillin, $100 \mathrm{mg} / \mathrm{ml}$ streptomycin and $1 \mathrm{mg} / \mathrm{ml}$ amphotericin B (all Gibco; Thermo Fisher Scientific, Inc.)] was then added to cover the explants. Cell culture dishes were left undisturbed at $37^{\circ} \mathrm{C}$ in a humidified incubator at $5 \% \mathrm{CO}_{2}$. The culture medium was replenished every 2 days until sporadic fibroblast-like cells grew from the tissue edge, usually after 5-7 days. Cells were passaged when cultures reached 80-90\% confluence. Third passage cells were used in experiments. HUMSCs at passage 3 were characterized using flow cytometry to examine the expression of pluripotent cell markers as previously detailed (15).

Chloromethylbenzamido-1,1'-dioctadecyl-3,3,3'3'-tetramethylindocarbocyanine perchlorate (CM-DiI; lipophilic carbocyanine dye) labelling (16). CM-DiI is a lipophilic carbocyanine fluorescent dye, which attaches to the cell membrane and has low cytotoxicity. CM-DiI can be well preserved after fixation, permeabilization and paraffin embedding of cells. Therefore, CM-Dil is been widely used for immunofluorescence staining on tissue sections (17). HUMSCs at passage 3 were cultured in complete growth medium under aforementioned conditions. Prior to labeling, all HUMSCs were suspended at a density of $1 \times 10^{6} / \mathrm{ml}$ in serum-free DMEM and incubated with $4 \mu \mathrm{MCM}-\mathrm{DiI}$ (Molecular Probes; Thermo Fisher Scientific, Inc.) for $15 \mathrm{~min}$ at $37^{\circ} \mathrm{C}$, followed by $10 \mathrm{~min}$ incubation at $4^{\circ} \mathrm{C}$ to optimize staining levels. Cells were then precipitated at $250 \mathrm{x}$ g for $5 \mathrm{~min}$ at $37^{\circ} \mathrm{C}$. Cell pellets were gently re-suspended in $37^{\circ} \mathrm{C}$ medium and washed twice. The efficiency of CM-DiI labeling was checked by immunofluorescence using fluorescence microscopy (Olympus BX51, Olympus Corporation, Tokyo, Japan). Briefly, the cells seeded onto coverslips and fixed in $4 \%$ paraformaldehyde for $30 \mathrm{~min}$ and then washed with PBS three times. Cells were counterstained with 4',6-diamidino-2-phenylindole (DAPI) for $5 \mathrm{~min}$ as a nuclear stain. Positive cells were scored from four randomly selected fields per specimen, and the efficiency of CM-DiI labeling was represented as incorporation into the positive rate. Viability of CM-DiI labeled HUMSCs was assessed by Trypan blue staining.

Preparation of acellular amnion. The preparation of acellular amnion was performed as detailed previously (18). Briefly, human amnion collected at cesarean section was washed with PBS under sterile conditions and stored at $-80^{\circ} \mathrm{C}$ in PBS containing $12 \%$ dimethyl sulfoxide. The amnion was frozen and thawed 3 times and cut into pieces $(2.5 \times 2.5 \mathrm{~cm})$, followed by the removal of epithelial cells by incubation in $0.02 \%$ EDTA at $37^{\circ} \mathrm{C}$ for $2 \mathrm{~h}$ using gentle scraping under a microscope. The spongy layer was also removed. The complete removal of the epithelial cells was confirmed by hematoxylin and eosin (H\&E) staining.

Extraction of PPP. PPP was the residual material of platelet rich plasma (PRP) therapy collected following cosmetic surgery procedures on outpatients of the Second Affiliated Hospital, Shantou University Medical College. Informed consent was obtained from the patients who provided PPP before the 
collection procedure. The specific extraction method was performed as previously described (19). Blood samples were centrifuged twice to obtain PPP and PRP. A $30 \mathrm{ml}$ venous blood sample was collected under aseptic conditions at room temperature, and was aspirated with a $21 \mathrm{G}$ needle into a $50 \mathrm{ml}$ sterile centrifuge tube preloaded with $6.5 \mathrm{ml}$ anticoagulant citrate dextrose solution. The blood sample was centrifuged for $15 \mathrm{~min}$ at $320 \mathrm{x} \mathrm{g}$, at $4^{\circ} \mathrm{C}$, resulting in the following three layers: An inferior layer composed of red cells, an intermediate layer composed of white cells, and a superior layer made up of plasma. The $20 \mathrm{ml}$ plasma layer was centrifuged for a further $5 \mathrm{~min}$ at 1,000 $\mathrm{x} \mathrm{g}$ in order to obtain a two-part plasma sample: The upper part, consisting of $18 \mathrm{ml} \mathrm{PPP}$; and the lower part, consisting of $2 \mathrm{ml}$ PRP. The PPP was gently aspirated with a pipette and placed in a sterile centrifuge tube, without being mixed with PRP. Allergic reactions were not observed when administering PPP in these experiments.

Preparation of PPPA and generation of wound animal model. When passage 3 CM-DiI labeled HUMSCs reached $80 \%$ confluence, HUMSCs were detached with trypsin solution, washed with $\mathrm{PBS}\left(37^{\circ} \mathrm{C}\right)$ and precipitated. Cell pellets were re-suspended in PPP at a density of $1 \times 10^{6} / \mathrm{ml}$, and $1 \mathrm{ml} \mathrm{PPP}$ was transferred to each well of a 12-well tissue culture plate (Corning Incorporated, Corning, NY, USA), followed by addition of $10 \%$ calcium gluconate at a volume ratio of 1:7. Preliminary experiments revealed that this ratio was the most conducive to forming PPP gels (data not shown). Plates were left undisturbed for $15 \mathrm{~min}$ at $37^{\circ} \mathrm{C}$ in a humidified incubator with 5\% $\mathrm{CO}_{2}$ to allow PPP gel formation. Following gel formation, fresh growth medium was added to cover the gel to maintain HUMSC growth. Gels containing HUMSCs were used in wound healing in animals the following day. Blank gels were made in the same way, without the inclusion of HUMSCs, and were used as a control. At the time of surgery, all gels were detached from the 12-well plates with forceps (Fig. 1A).

As HUMSCs possess immunosuppressive properties, Sprague-Dawley (SD) rats were used rather than severe immune deficient mice. SD rats (age, 16 weeks; weight, 240-260g; n=36) were purchased from Shantou University Medical College Laboratory Animal Center (SUMC; Shantou, China) and maintained under specific pathogen free conditions in the Laboratory Animal Center of SUMC. All animals were allowed to acclimate for 1 week in the facility before experiments were performed. Animal protocols were approved by the Institutional Animal Care and Use Committee at SUMC.

SD rats were randomly assigned into four groups ( $\mathrm{n}=9$ rats/group): Group 1, grafted with PPPA+HUMSCs (PPPA+cells; PPPAC); group 2, grafted with PPPA (PPPA); group 3, injected with HUMSCs (Injection); and group 4, injected with PBS as blank control (Blank). An excisional wound splinting mouse model was generated as previously described (20) with slight modifications. In brief, after hair removal from the dorsal surface under anesthesia (10\% chloral hydrate solution; $0.1 \mathrm{ml} / 20 \mathrm{~g}$; Sigma-Aldrich; Merck KGaA, Darmstadt, Germany), two $14 \mathrm{~mm}$ (area $1.5386 \mathrm{~cm}^{2}$ ) full thickness excisional skin wounds were created on each side of the midline. A donut-shaped silicone splint was placed so that the wound was centered within the splint, which was fixed to the skin by an immediate-bonding adhesive. Interrupted sutures were utilized to stabilize its position. The same shaped hoops were attached to the silicone splint to prevent rats from gnawing it. Acellular amnion was placed over the wounds. After the surgical procedure, the animals were housed individually. Our preliminary studies suggested that the adhesive on the skin in rats prior to the experiment did not cause any skin irritation or allergic reaction. Each wound in the PPPAC group was grafted with PPP gel with $1 \mathrm{x}^{6} \mathrm{CM}$-DiI labeled HUMSCs (Fig. 1B-D). In the PPPA group, the same procedure was employed for the PPPAC group, except for the use of the blank gels. Each wound in the Injection Group received 1,000 $\mu 1$ HUMSC suspension containing $1 \times 10^{6} \mathrm{CM}-\mathrm{DiI}-$ labeled HUMSCs, among which $800 \mu \mathrm{l}$ was used for subcutaneous injection around the wound and $200 \mu \mathrm{l}$ for topical application on the wound bed. Blank Group animals were injected with PBS as a blank control.

Wound healing assessment. Following surgery, wound dressings were changed every day and the behavioral activity and wound healing progression of the SD rats was monitored. On days $0,2,4,6,8,10,12$ and 14 post-surgery, the open wounds on both sides of midline of each animal from the four groups were imaged and wound healing was assessed by comparison of images captured at different time points with the initial images using an image analyzer (Image Pro Plus 6, Media Cybernetics, Inc., Rockville, MD, USA). The healing index included re-epithelialization and contraction. The healing rate was calculated as (area of original wound-area of left exposing wound)/area of original wound $\times 100 \%$.

In addition, $2 \mathrm{SD}$ rats randomly selected from each group were sacrificed at 3, 7 or 14 days, and the skin samples containing the wound and $3 \mathrm{~mm}$ of the surrounding skin were harvested (21), fixed in 10\% buffered formalin and embedded in paraffin. $\mathrm{H} \& \mathrm{E}$ staining was performed on $3-\mu \mathrm{m}$ thick tissue sections.

Determination of the HUMSC distribution in the wound. Sequential slides were used for detecting the distribution of CM-DiI- labeled HUMSCs by immunofluorescence. Briefly, the sections were rehydrated and the antigens were retrieved via microwaving in $10 \mathrm{mM}$ sodium citrate $(\mathrm{pH} \mathrm{6.0)}$ for $10 \mathrm{~min}$, followed by counterstaining with DAPI. Following this, slides were washed 3 times with PBS, covered with anti-fade solution, and positive cells were scored on the wound region under fluorescence microscopy to evaluate the distribution of HUMSCs.

Determination of expression levels of growth factors in HUMSCs in PPPA. The same procedure described above was used to generate PPPA. In the experimental group (PPPAC group), passage 3 PPPA HUMSCs (a total of $1 \times 10^{6} \mathrm{CM}-\mathrm{DiI}$ labeled HUMSCs in $1 \mathrm{ml} \mathrm{PPP}$ ) grown in a 12-well plate, were cultured in vitro, and the supernatant of each well in experimental group was collected on days 1, 3, 7 and 14. In the control group (PPPA group), PPPA without HUMSCs formed in a 12-well plate were cultured in vitro, and the supernatant was collected on day 1 as an initial reference. A monolayer group (a total of $1 \times 10^{6} \mathrm{CM}$-DiI labeled HUMSCs in 2D culture conditions in 1 well of a 12-well plate) were used as a $2 \mathrm{D}$ reference (2D group). The culture medium was changed 
A

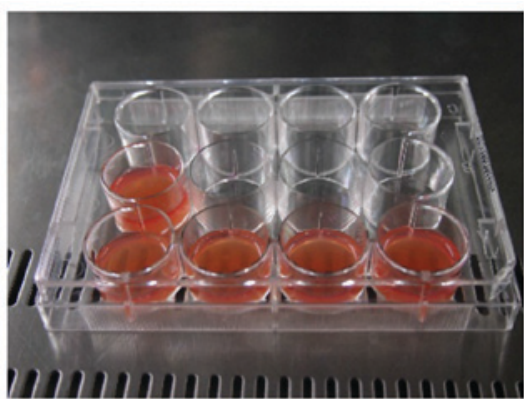

C

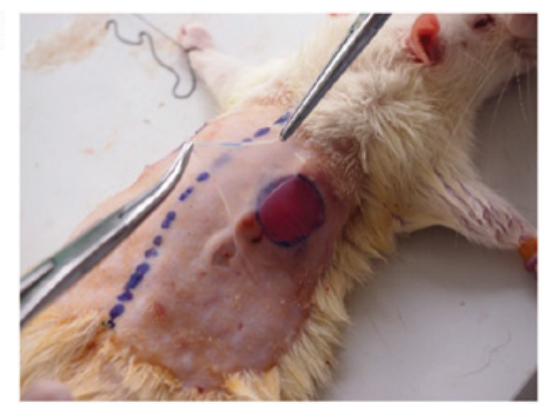

B

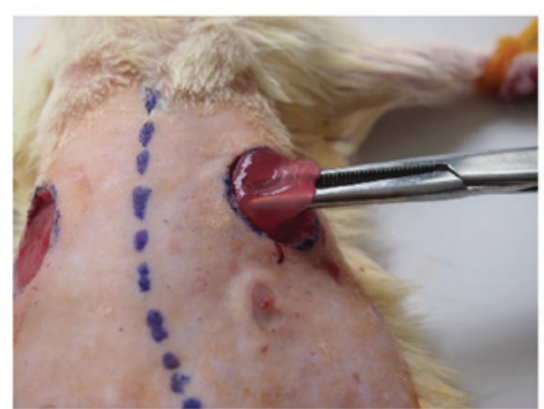

D

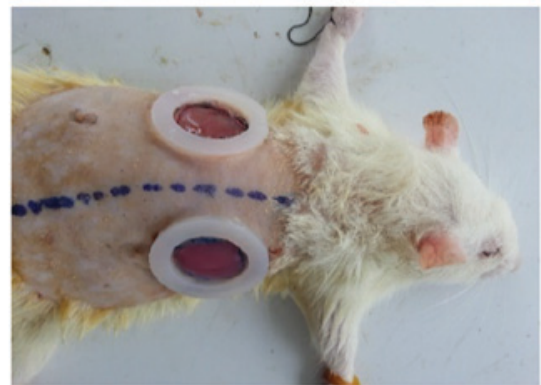

Figure 1. Generation of full-thickness excisional skin wounds and preparation of HUMSCs in PPP gel. (A) HUMSCs in PPP gel. (B) Once the full-thickness excisional skin wounds were generated, PPP gel with or without $1 \times 10^{6}$ chloromethylbenzamido-1,1'-dioctadecyl-3,3,3'3'-tetramethylindocarbocyanineperchlorate labeled HUMSCs inside (PPPAC/PPPA group) was used to cover the dorsal skin wounds surface. (C) Acellular amnion was used to cover the PPP gel in the dorsal skin wounds. (D) A donut-shaped silicone splint was placed so that the wound was centered within the splint. PPP, platelet poor plasma; PPPAC, platelet poor plasma gel combined with amnion grafted with human umbilical cord-derived mesenchymal stem cells; HUMSCs, human umbilical cord-derived mesenchymal stem cells.

$24 \mathrm{~h}$ prior to supernatant collection, and the expression levels of growth factors including insulin-like growth factor (IGF-1), hepatocyte growth factor (HGF), transformation growth factor (TGF)- $\beta 1$, vascular endothelial growth factor (VEGF) and keratinocyte growth factor (KGF) in the collected supernatant was detected using ELISA kits (Human VEGF Quantikine ELISA kit; cat. no. SVE00; Human HGF Quantikine ELISA kit; cat. no. SHG00; Human KGF Quantikine ELISA kit; cat. no. DKG00; Human TGF- $\beta 1$ Quantikine ELISA kit; cat. no. SB100B; and Human IGF-1 Quantikine ELISA kit; cat. no. SG100) from R\&D Systems, Inc. (Minneapolis, MN, USA) according to the manufacturer's protocol. The data were compiled from three independent assays per sample with each performed in duplicate.

Statistical analysis. Data were analyzed using SPSS version 20.0 (IBM Corp., Armonk, NY, USA) and are presented as the mean \pm standard deviation. Student's paired t-test was performed for comparison of data of paired samples. One-way analysis of variance was used for multiple group comparisons followed by the least significant differences t-test. $\mathrm{P}<0.05$ was considered to indicate a statistically significant difference.

\section{Results}

Isolation of HUMSCs from human umbilical cords. Fig. 2 presents the primary culture of isolated HUMSCs. At day 5-7 in culture, spindle-shape cells (Fig. 2A, black arrow) began to migrate out from Wharton's jelly fragments (Fig. 2A, white arrow). Half of the volume of culture medium was replaced with new fresh medium every 3-4 days until cells completely covered the culture area, which was reached by $\sim$ day 10-14.
HUMSCs exhibited fibroblast-like morphology, with most demonstrating a flat, wide and polygonal appearance after passaging. The above-mentioned morphology did not alter significantly up to passage 9 (Fig. 2B-D). Cells at passage 3 expressed cluster of differentiation (CD)29, CD59 and CD44, but not CD34 and human leukocyte antigen D related (data not shown), and were used in the subsequent experiments.

CM-DiI efficiently labels isolated HUMSCs. CM-DiI labeled 98.4\% HUMSCs isolated from human umbilical cord as determined by immunofluorescence (Fig. 3), while only $<5 \%$ CM-DiI-positive HUMSCs were trypan blue positive (17) (data not shown). Further culture revealed that CM-DiI labeling, present in the cytoplasm and not the nucleus, did not significantly affect the proliferation of HUMSCs (data not shown). CM-DiI-labeled fluorescence was clearly visible at 14 day after culture.

PPPA improves HUMSC-mediated wound healing in rats with full thickness excisional skin wounds. In the animal wound model used in this study, it was demonstrated that PPPA significantly enhanced HUMSC-mediated wound healing in SD rats (Table I). From day 4 post-surgery, the healing rate of the PPPAC group was significantly higher compared with the other three experimental groups (Table I; $\mathrm{P}<0.05$ and $\mathrm{P}<0.01$ ). However, at days 2, 6, 8 and 14, the difference in the healing rate between the PPPA group and the Injection group was not statistically significant (Table I). Wound contraction was markedly reduced in the PPPAC and PPPA groups compared with the injection and blank groups (Fig. 4A). Furthermore, the thickness of the newly formed epidermis layer of the PPPAC group grew faster to cover the wounded skin tissue compared 
Table I. Wound healing rate of Sprague-Dawley rats ( $\mathrm{n}=9 /$ group).

Wound healing rate $(\%)$

\begin{tabular}{lccccccc}
\hline Group & Day 2 & Day 4 & Day 6 & Day 8 & Day 10 & Day 12 & Day 14 \\
\hline Blank & $9.01 \pm 2.17$ & $17.15 \pm 2.84$ & $26.14 \pm 3.75$ & $45.52 \pm 4.02$ & $64.26 \pm 4.41$ & $83.27 \pm 2.99$ & $90.10 \pm 2.86$ \\
Injection & $9.73 \pm 2.45$ & $19.06 \pm 2.98$ & $32.48 \pm 4.89$ & $55.68 \pm 7.61$ & $73.55 \pm 4.86$ & $86.05 \pm 3.68$ & $94.67 \pm 2.63$ \\
PPPA & $11.46 \pm 1.86^{\mathrm{a}}$ & $22.81 \pm 2.92^{\mathrm{b} . \mathrm{c}}$ & $36.50 \pm 4.68^{\mathrm{b}}$ & $57.53 \pm 3.15^{\mathrm{b}}$ & $80.12 \pm 3.83^{\mathrm{b}, \mathrm{c}}$ & $91.50 \pm 2.09^{\mathrm{b}, \mathrm{c}}$ & $96.53 \pm 1.41^{\mathrm{b}}$ \\
PPPAC & $11.11 \pm 3.06$ & $25.45 \pm 4.69^{\mathrm{b}-\mathrm{d}}$ & $46.35 \pm 6.51^{\mathrm{b}-\mathrm{d}}$ & $65.02 \pm 6.12^{\mathrm{b}-\mathrm{d}}$ & $85.10 \pm 3.56^{\mathrm{b}-\mathrm{d}}$ & $95.50 \pm 2.10^{\mathrm{b}-\mathrm{d}}$ & $99.51 \pm 0.69^{\mathrm{b}-\mathrm{d}}$ \\
\hline
\end{tabular}

Data are presented as the mean \pm standard deviation. ${ }^{a} \mathrm{P}<0.05$, ${ }^{b} \mathrm{P}<0.01$ vs. Blank, ${ }^{\mathrm{c}} \mathrm{P}<0.05$ vs. Injection, ${ }^{\mathrm{d}} \mathrm{P}<0.05$ vs. PPPA. PPPA, platelet poor plasma gel combined with amnion; PPPAC, platelet poor plasma gel combined with amnion grafted with human umbilical cord-derived mesenchymal stem cells.
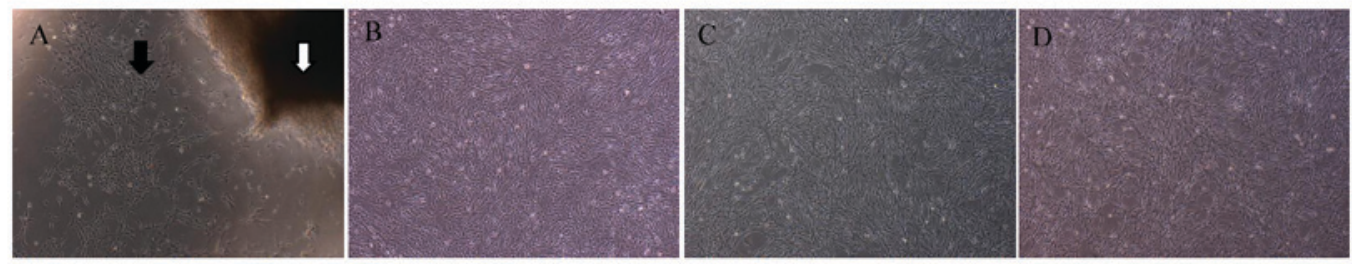

Figure 2. Morphology of primary cultured HUMSCs. (A) Primary cultured HUMSCs at day 7. The tissue was adherent (white arrow) and cells migrated out from adherent tissue of Wharton's jelly (black arrow). The growing HUMSCs exhibited a fibroblast-like shape, and arranged as whirl pool. Cells at passage (B) 1, (C) 3 and (D) 9. Magnification, x40. HUMSCs, human umbilical cord-derived mesenchymal stem cells.
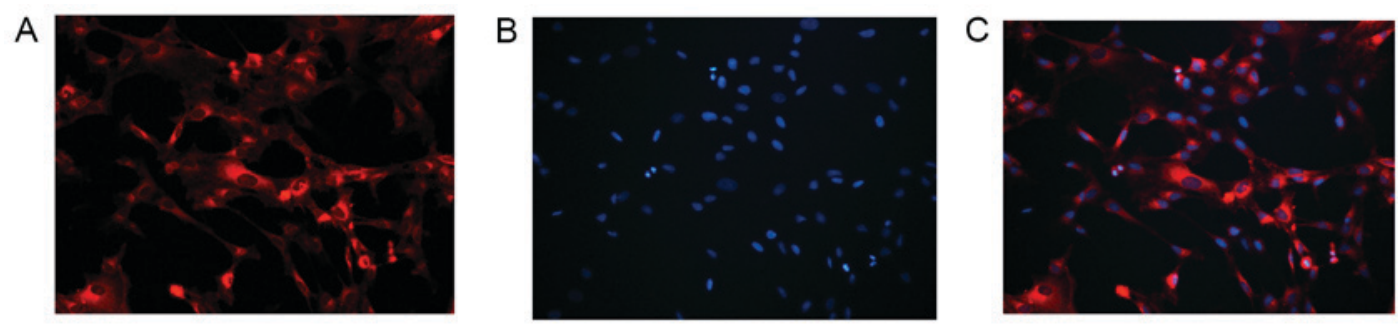

Figure 3. CM-DiI-labeled human umbilical cord-derived mesenchymal stem cells. (A) cytoplasmic but not nuclear labeling with CM-DiI. (B) Nuclear counterstaining with 4',6-diamidino-2-phenylindole. (C) The merge of (A) and (B). Magnification, x200. CM-DiI, chloromethylbenzamido-1,1'-dioctadecyl-3,3,3'3'-tetramethylindocarbocyanine perchlorate.

with the other groups either on the 7th or the 14th day, as examined by histology. In addition, the dermal papillae and the amount of CM-DiI-labeled HUMSCs in the regenerated skin tissue were significantly increased in the PPPAC group. The alignment of fibers in the healing skin tissue appeared more regular in the PPPAC group than the other groups (Fig. 4B). Therefore, it was concluded that the PPPAC group demonstrates superior wound healing compared with the other three groups.

Distribution of CM-DiI-labeled HUMSCs in the newly formed healing skin tissue. Next, the present study determined the distribution of CM-DiI labeled HUMSCs in the wounded area of skin of rats from the different experimental groups. Immunofluorescence was observed in the basal layer of epidermis, dermis, spinous layer and superficial fascia in newly regenerated skin tissue in the PPPAC and Injection groups (Fig. 5 and data not shown). Fluorescent-positive cells were polygonal or spherical in shape and were located in the newly regenerated skin tissue or in the nearby healthy skin tissue. The distribution of HUMSCs in PPPAC group was more homogeneous compared with that in PPPA group. Taken together, these results indicated that the PPPAC group demonstrates a better survival rate of HUMSCs after engrafting.

Increased levels of HUMSC-secreted growth factors at different time points in the PPPAC group. ELISAs were subsequently performed to evaluate the protein expression levels of secreted growth factors (including HGF, TGF- $\beta_{1}$, VEGF, IGF-1 and KGF) in HUMSCs at different culture stages and times in the PPPA group (Fig. 6). As PPPA contained certain baseline levels of growth factors, the culture medium was collected at day 1 from the PPPA group as an initial reference value. The findings suggested that the secretion levels of HGF, TGF- $\beta_{1}$, VEGF, IGF- 1 and KGF released by HUMSCs in the PPPAC group were significantly increased compared 

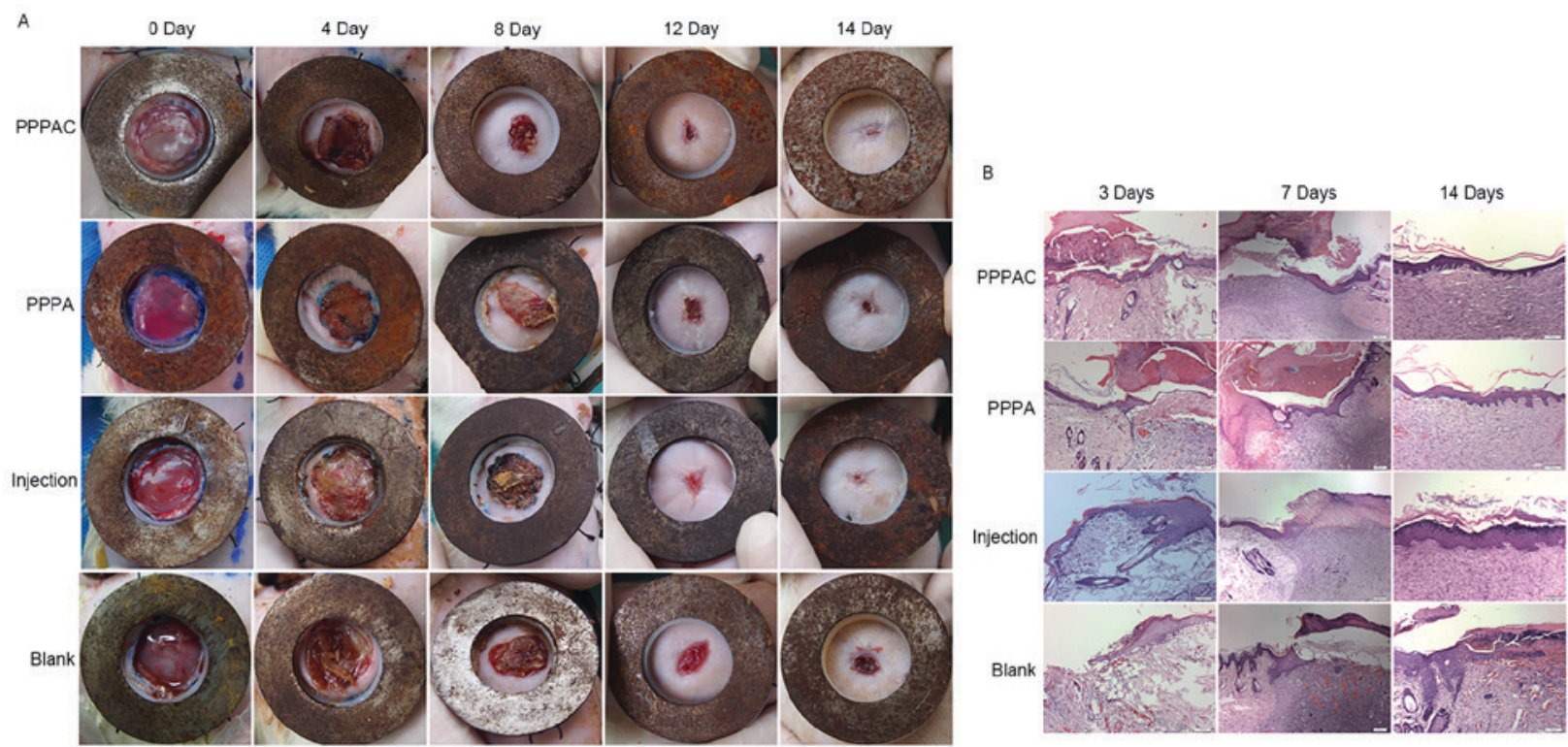

Figure 4. Characterization of wound healing. (A) Representative images of wounds at the indicated time points after surgery. (B) Histological examination of wound healing in the different groups. The biopsies of cutaneous wound healed tissues were examined on day 3, 7, 14 after operation. The newly formed epidermis layer of the PPPAC group grew faster and thicker to cover the skin tissue defect wound than that of the other groups. PPPAC treatment-induced regenerating tissue exhibited significantly increased dermal papillae and the number of cells. Day 3, scale bar=100 $\mu$ m; magnification, x100. Day 7, scale bar $=200 \mu \mathrm{m}$; magnification, $\mathrm{x} 40$. Day 14, scale bar=100 $\mu \mathrm{m}$; magnification, $\mathrm{x} 100$. PPPAC, platelet poor plasma gel combined with amnion grafted with human umbilical cord-derived mesenchymal stem cells.

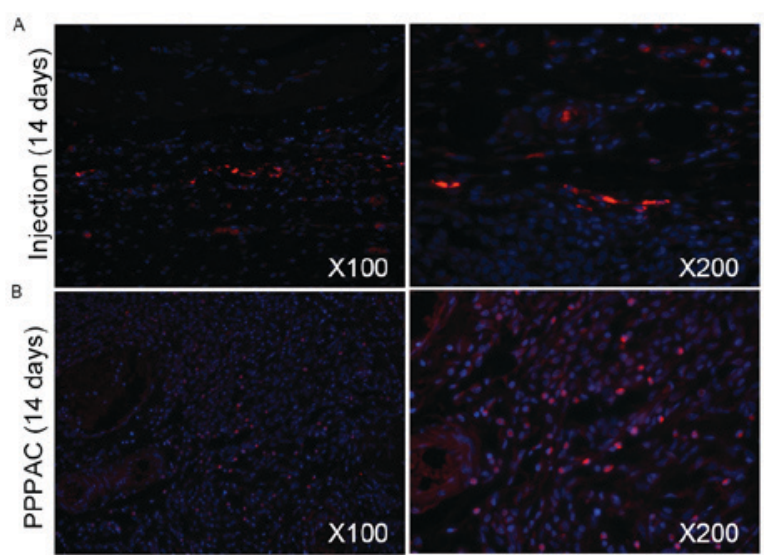

Figure 5. Examination of distribution of CM-DiI-labeled HUMSCs in the healed wound. At days 7 and 14, a biopsy was performed to obtain the healed wound tissues, which were fixed and sectioned, followed by immunofluorescence examination for distribution of CM-DiI-labeled HUMSCs. Red fluorescence indicates positive cells, blue fluorescence is nuclear staining with 4',6-diamidino-2-phenylindole. The positive cells were emitting red fluorescence as indicated in the images. Representative images at day 14 from the (A) Injection and (B) PPPA groups. CM-DiI, chloromethylbenzamido-1,1'-dioctadecyl-3,3,3'3'-tetramethylindocarbocyanine perchlorate HUMSCs, human umbilical cord-derived mesenchymal stem cells; PPPA, platelet poor plasma gel combined with amnion.

with those from the 2D group. Therefore, it was concluded that PPPAC promotes the release of growth factors from HUMSCs after engrafting into the wounded area of skin.

\section{Discussion}

Previously, several studies have isolated MSCs from umbilical cord blood (22-24). However, Secco et al (25) reported that more MSCs were present in the cord itself than in the blood, and that different experimental conditions used to isolate/culture MSCs accounted for the differences in experimental outcomes and efficacy of MSCs. The present study followed a previously published protocol and isolated and cultured stem cells from human umbilical cord Wharton's jelly (26), and successfully purified HUMSCs from five samples. HUMSCs were isolated and characterized, and it was demonstrated that i) isolated HUMSCs highly express CD29, CD44 and CD59, and ii) isolated HUMSCs are able to differentiate into multiple cell types including adipocytes and osteoblasts under appropriate conditions, as previously reported $(27,28)$. Furthermore, the HUMSCs displayed fibroblast-like morphology, and most were flat, wide and polygonal following passaging. These properties did not alter significantly up to passage 9 . Therefore, a sufficient number of cells were obtained to perform experiments.

Compared with stem cells from other sources such as ESCs, HUMSCs may prove a more suitable choice for clinical applications with respect to ethical and practical issues. Clinical trials have used MSCs to treat human diseases, including Parkinson's disease and myocardial infarction (29). However, their use regarding their application in wound healing remains unknown. Several barriers to their clinical use exist, including poor integration of MSCs grafted into the wound. One of the primary mechanisms by which MSCs exert their therapeutic effects is through paracrine activity, which is affected by many factors including local microenvironment, inflammatory cytokines and cell phenotypes. A growing body of evidence suggests that when differentiated cells reach confluence, they cease to proliferate due to contact inhibition. These contact-inhibited cells exhibit lower protein synthesis and metabolic rate $(30,31)$. In previous studies, MSCs were expanded on tissue culture plastic as monolayers in vitro, and lost their cell-specific properties with several rounds of 

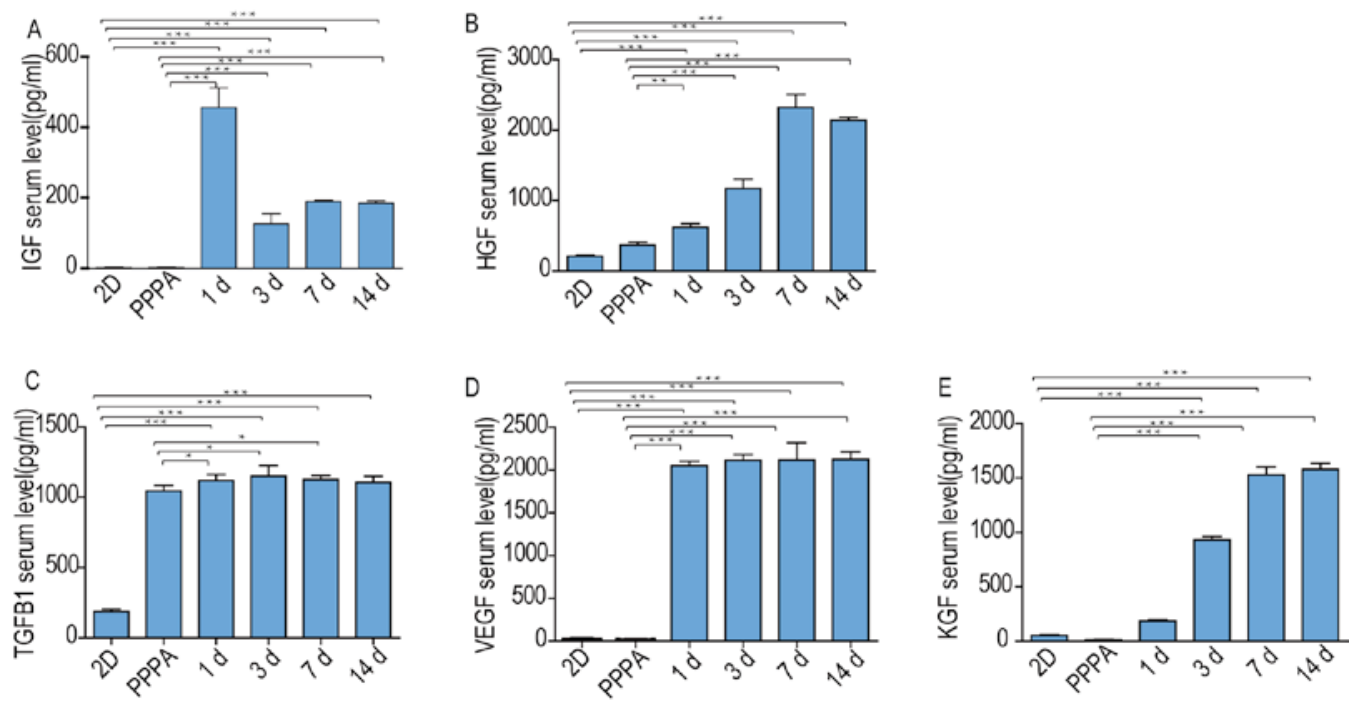

Figure 6. Measurement of levels of growth factors released by human umbilical cord-derived mesenchymal stem cells at different time points. (A) IGF-1, (B) HGF, (C) TGF- $\beta 1$, (D) VEGF and (E) KGF serum levels were measured at days 1, 3, 7 and 14 following gel formation. The culture medium in the PPPA group was collected at day 1 as an initial reference. The culture medium from the $2 \mathrm{D}$ group (monolayers group) was collected as the $2 \mathrm{D}$ reference. ${ }^{*} \mathrm{P}<0.05$, ${ }^{* *} \mathrm{P}<0.01,{ }^{* * *} \mathrm{P}<0.001$. IGF-1, including insulin-like growth factor; HGF, hepatocyte growth factor; TGF- $\beta 1$, transformation growth factor- $\beta 1$; VEGF, vascular endothelial growth factor; KGF, keratinocyte growth factor; PPPA, platelet poor plasma gel combined with amnion.

passages and prolonged cultured time in vitro (32). In contrast, MSCs developed in 3D conditions using AggreWell expressed genes associated with angiogenesis and wound healing, and exhibited better multipotent differentiation capacity in vitro (33).

The findings of the present study suggested that HUMSCs cultured in PPPAC upregulated the secretion of paracrine molecules such as HGF, TGF- $\beta_{1}$, VEGF, IGF-1 and KGF, involved in cellular proliferation and wound re-epithelialization, compared to the monolayer culture of HUMSCs. These findings were in line with those previously reported for MSCs cultured in 3D conditions using AggreWell (5).

In recent years, PRP has been widely used in plastic surgery $(34,35)$. Although during the extraction process, centrifuge parameters were adjusted to obtain better PRP, the amount of discarded PPP remained large (36). Therefore, PPP was selected as the cell carrier and scaffold material due to easy acquisition. In future clinical applications, PPP is able to be obtained from the blood of the patient, autologous material without immunogenicity, and is available in sufficient quantities. PPP has been demonstrated to contain a large amount of fibrinogen, and PPP solidification occurs after calcium gluconate is added. In this coagulation process, fibrinogen is converted into fibrin (37), which forms a mesh structure inside the PPP gel and provides3D culture conditions for HUMSCs and promotes wound scabbing. These conditions improved the efficiency of HUMSCs in grafting, increasing HUMSC adhesion (38) and delivering better functional interactions between HUMSCs (39). The PPP gel in the present experiment was a colloidal semi-solid gel, easy desiccate and easy to infect. Therefore, this was combined with amnion (PPPA), using acellular amnion to seal the gel and wound. Amnion is a tissue of interest as a biological dressing due to its biological properties and immunologic characteristics (40). This PPPA combination is easy to make with moderate costs, easily adheres to wounds and can effectively close wounds in the early trauma period, thus reducing the bacterial infection. In the present study, 14 days after surgery, the wound healing rate of the PPPAC group was significantly higher than the other experimental groups. In addition, it was demonstrated that wound contraction in the PPPAC group was inhibited more efficiently than other groups. Furthermore, using immunofluorescence, CM-DiI labeled HUMSCs were identified to be scattered throughout the basal layer of the epidermis, dermis, spinous layer, superficial fascia and connective tissue around the wound. At particular time points examined, it was observed that the amount of CM-DiI-labeled HUMSCs in the PPPAC group was much higher than that of the other three experimental groups. Taken together, these findings demonstrated that the PPPAC group has the highest grafting survival rate and healing capacity among all groups examined.

Skin wound repair is an intricate and highly coordinated process that generally can be divided into three overlapping but distinct phases: The vascular and inflammatory phase, the granulation tissue formation, re-epithelialization and proliferative phase, and the remodeling phase (41). Interruption of one or more phases of this process can result in chronic wound healing, fibrosis or scarring. Due to the lack of epidermal basal and dermal layers, it is difficult to rebuild normal structures in full-thickness excisional skin wounds. In H\&E sections at day 3 following grafting, wounds in each group seeped out obviously with infiltration with neutrophils, macrophages and other inflammatory cells, and the remaining epidermal stem cells migrated from the wound edges to the wound center. In the Injection and Blank groups, due to the deficiency of dermal layer and subcutaneous tissue, sufficient granulation did not occur; therefore, epidermal stem cells were unable to migrate to cover the surface of the wound. Cells accumulated and 
proliferated at the margin surrounding the wound, as examined at days 7 and 14 after grafting. Re-epithelialization rates in these two groups were much slower than that of the PPPAC group. In contrast, in the PPPAC and PPPA groups, inflammation was mild and epidermal stem cells migrated a longer distance. It was also demonstrated that the application of PPPAC facilitated the formation of the epidermis layer in the repairing skin tissue with thickness similar to the surrounding normal skin, increasing the amount of dermal ridges, and increasing the alignment of fibers to a more regular morphology. These observations suggested that the administration of PPPAC was not only able to accelerate the speed of wound healing, but also improved the quality of the wound healing.

To further understand the molecular basis underlying the increased rate of wound healing achieved by PPPAC, the levels of growth factors in the culture mediums of these $3 \mathrm{D}$ and 2D culture conditions were measured and compared, and the secretion levels of HGF, TGF- $\beta_{1}$, VEGF, IGF-1 and KGF released by HUMSCs in the PPPAC group were significantly higher in 3D culture conditions compared with those in the 2D group. As these cytokines were involved in re-epithelialization, neovascularization and remodeling of wound healing, it was hypothesized that increased secretion of these cytokines may serve a role in facilitating wound healing achieved by PPPAC.

In conclusion, the present study demonstrated that PPPA modifies the paracrine activities of HUMSCs, provides a relatively stable grafting environment and improves the efficiency of HUMSC in wound healing in the animal model used in this study. Further studies are required to investigate the specific mechanisms by which PPPAC promotes wound healing, demonstrating its potential utility for clinical therapeutic applications in skin grafts and wound healing.

\section{Acknowledgements}

The present study was supported by the National Natural Science Foundation of China (grant no. 81272116) and the Scientific Research Foundation for the Returned Overseas Chinese Scholars, Ministry of Education of China (grant no. [2013] 693).

\section{References}

1. Atiyeh BS, Gunn SW and Hayek SN: State of the art in burn treatment. World J Surg 29: 131-148, 2005.

2. Hocking AM and Gibran NS: Mesenchymal stem cells: Paracrine signaling and differentiation during cutaneous wound repair. Exp Cell Res 316: 2213-2219, 2010.

3. Prockop DJ: Marrow stromal cells as stem cells for nonhematopoietic tissues. Science 276: 71-74, 1997.

4. da Silva Meirelles L, Chagastelles PC and Nardi NB: Mesenchymal stem cells reside in virtually all post-natal organs and tissues. J Cell Sci 119: 2204-2213, 2006.

5. Bongso A and Fong CY: The therapeutic potential, challenges and future clinical directions of stem cells from the Wharton's jelly of the human umbilical cord. Stem Cell Rev 9: 226-240, 2013.

6. Jadalannagari S and Aljitawi OS: Ectodermal Differentiation of wharton's jelly mesenchymal stem cells for tissue engineering and regenerative medicine applications. Tissue Eng Part B Rev 21: 314-322, 2015.

7. Zhao G, Liu F, Lan S, Li P, Wang L, Kou J, Qi X, Fan R, Hao D, $\mathrm{Wu} \mathrm{C}$, et al: Large-scale expansion of Wharton's jelly-derived mesenchymal stem cells on gelatin microbeads, with retention of self-renewal and multipotency characteristics and the capacity for enhancing skin wound healing. Stem Cell Res Ther 6: 38, 2015
8. Horwitz EM, Le Blanc K, Dominici M, Mueller I, Slaper-Cortenbach I, Marini FC, Deans RJ, Krause DS and Keating A; International Society for Cellular Therapy: Clarification of the nomenclature for MSC: The International Society for Cellular Therapy position statement. Cytotherapy 7 : 393-395, 2005.

9. Tang Q, Chen Q, Lai X, Liu S, Chen Y, Zheng Z, Xie Q, Maldonado M, Cai Z, Qin S, et al: Malignant transformation potentials of human umbilical cord mesenchymal stem cells both spontaneously and via 3-methycholanthrene induction. PLoS One 8: e81844, 2013

10. Hanson SE, Bentz ML and Hematti P: Mesenchymal stem cell therapy for nonhealing cutaneous wounds. Plast Reconstr Surg 125: 510-516, 2010.

11. Balaji S, Keswani SG and Crombleholme TM: The role of mesenchymal stem cells in the regenerative wound healing phenotype Adv Wound Care (New Rochelle) 1: 159-165, 2012.

12. Sasaki M, Abe R, Fujita Y, Ando S, Inokuma D and Shimizu H: Mesenchymal stem cells are recruited into wounded skin and contribute to wound repair by transdifferentiation into multiple skin cell type. J Immunol 180: 2581-2587, 2008.

13. Khosrotehrani K: Mesenchymal stem cell therapy in skin: Why and what for? Exp Dermatol 22: 307-310, 2013.

14. Arno AI, Amini-Nik S, Blit PH, Al-Shehab M, Belo C, Herer E, Tien $\mathrm{CH}$ and Jeschke MG: Human Wharton's jelly mesenchymal stem cells promote skin wound healing through paracrine signaling. Stem Cell Res Ther 5: 28, 2014.

15. Huang P, Lin LM, Wu XY, Tang QL, Feng XY, Lin GY, Lin X, Wang HW, Huang TH and Ma L: Differentiation of human umbilical cord Wharton's jelly-derived mesenchymal stem cells into germ-like cells in vitro. J Cell Biochem 109: 747-754, 2010.

16. Weir C, Morel-Kopp MC, Gill A, Tinworth K, Ladd L, Hunyor SN and Ward C: Mesenchymal stem cells: Isolation, characterisation and in vivo fluorescent dye tracking. Heart Lung Circ 17: 395-403, 2008.

17. Hemmrich K, Meersch M, von Heimburg D and Pallua N: Applicability of the dyes CFSE, CM-DiI and PKH26 for tracking of human preadipocytes to evaluate adipose tissue engineering. Cells Tissues Organs 184: 117-127, 2006.

18. Yang L, Shirakata Y, Shudou M, Dai X, Tokumaru S, Hirakawa S, Sayama K, Hamuro J and Hashimoto K: New skin-equivalent model from de-epithelialized amnion membrane. Cell Tissue Res 326: 69-77, 2006.

19. Woo SH, Kim JP, Park JJ, Chung PS, Lee SH and Jeong HS: Autologous platelet-poor plasma gel for injection laryngoplasty. Yonsei Med J 54: 1516-1523, 2013.

20. Chen L, Tredget EE, Wu PY and Wu Y: Paracrine factors of mesenchymal stem cells recruit macrophages and endothelial lineage cells and enhance wound healing. PLoS One 3: e1886, 2008 .

21. Galiano RD, Michaels J 5th, Dobryansky M, Levine JP and Gurtner GC: Quantitative and reproducible murine model of excisional wound healing. Wound Repair Regen 12: 485-492, 2004.

22. Hussain I, Magd SA, Eremin O and El-Sheemy M: New approach to isolate mesenchymal stem cell (MSC) from human umbilical cord blood. Cell Biol Int 36: 595-600, 2012.

23. Park SE, Jung NY, Lee NK, Lee J, Hyung B, Myeong SH, Kim HS, Suh YL, Lee JI, Cho KR, et al: Distribution of human umbilical cord blood-derived mesenchymal stem cells (hUCB-MSCs) in canines after intracerebroventricular injection. Neurobiol Aging 47: 192-200, 2016.

24. Ding Y, Yang H, Feng JB, Qiu Y, Li DS and Zeng Y: Human umbilical cord-derived MSC culture: The replacement of animal sera with human cord blood plasma. In Vitro Cell Dev Biol Anim 49: 771-777, 2013

25. Secco M, Zucconi E, Vieira NM, Fogaca LL, Cerqueira A, Carvalho MD, Jazedje T, Okamoto OK, Muotri AR and Zatz M: Multipotent stem cells from umbilical cord: Cord is richer than blood! Stem Cells 26: 146-150, 2008.

26. Ma L, Feng XY, Cui BL, Law F, Jiang XW, Yang LY, Xie QD and Huang TH: Human umbilical cord Wharton's Jelly-derived mesenchymal stem cells differentiation into nerve-like cells. Chin Med J (Engl) 118: 1987-1993, 2005.

27. Karahuseyinoglu S, Cinar O, Kilic E, Kara F, Akay GG, Demiralp DO, Tukun A, Uckan D and Can A: Biology of stem cells in human umbilical cord stroma: In situ and in vitro surveys. Stem Cells 25: 319-331, 2007

28. MacKenzie TC and Flake AW: Human mesenchymal stem cells: Insights from a surrogate in vivo assay system. Cells Tissues Organs 171: 90-95, 2002. 
29. Song H, Song BW, Cha MJ, Choi IG and Hwang KC: Modification of mesenchymal stem cells for cardiac regeneration. Expert Opin Biol Ther 10: 309-319, 2010.

30. Leontieva OV, Demidenko ZN and Blagosklonny MV: Contact inhibition and high cell density deactivate the mammalian target of rapamycin pathway, thus suppressing the senescence program. Proc Natl Acad Sci USA 111: 8832-8837, 2014.

31. Hayes CS, Koskiniemi S, Ruhe ZC, Poole SJ and Low DA: Mechanisms and biological roles of contact-dependent growth inhibition systems. Cold Spring Harb Perspect Med 4: pii: a010025, 2014.

32. Reiser J, Zhang XY, Hemenway CS, Mondal D, Pradhan L and La Russa VF: Potential of mesenchymal stem cells in gene therapy approaches for inherited and acquired diseases. Expert Opin Biol Ther 5: 1571-1584, 2005.

33. Michel M, L'Heureux N, Pouliot R, Xu W, Auger FA and Germain L: Characterization of a new tissue-engineered human skin equivalent with hair. In Vitro Cell Dev Biol Anim 35: 318-326, 1999.

34. Nita AC, Orzan OA, Filipescu M and Jianu D: Fat graft, laser $\mathrm{CO}_{2}$ and platelet-rich-plasma synergy in scars treatment. J Med Life 6: 430-433, 2013.

35. Kawazoe T and Kim HH: Tissue augmentation by white blood cell-containing platelet-rich plasma. Cell Transplant 21: 601-607, 2012 .
36. Amable PR, Carias RB, Teixeira MV, da Cruz Pacheco I, Corrêa do Amaral RJ, Granjeiro JM and Borojevic R: Platelet-rich plasma preparation for regenerative medicine: Optimization and quantification of cytokines and growth factors. Stem Cell Res Ther 4: 67, 2013

37. Diamond SL: Systems biology of coagulation. J Thromb Haemost 11 (Suppl 1): S224-S232, 2013.

38. Sierra DH: Fibrin sealant adhesive systems: A review of their chemistry, material properties and clinical applications. J Biomater Appl 7: 309-352, 1993.

39. Malafaya PB, Silva GA and Reis RL: Natural-origin polymers as carriers and scaffolds for biomolecules and cell delivery in tissue engineering applications. Adv Drug Deliv Rev 59: 207-233, 2007.

40. Insausti CL, Alcaraz A, García-Vizcaíno EM, Mrowiec A López-Martínez MC, Blanquer M, Piñero A, Majado MJ, Moraleda JM, Castellanos G and Nicolás FJ: Amniotic membrane induces epithelialization in massive posttraumatic wounds. Wound Repair Regen 18: 368-377, 2010.

41. Gurtner GC, Werner S, Barrandon Y and Longaker MT: Wound repair and regeneration. Nature 453: 314-321, 2008. 